\title{
ON ADAPTIVE OPTIMAL INPUT DESIGN
}

J.D. Stigter*, D. Vries*, K.J. Keesman*

\author{
*Systems and Control Group \\ Wageningen University and Research Center \\ Mansholtlaan 10-12 \\ 6708 PA Wageningen \\ The Netherlands \\ e-mail: hans.stigter@wur.nl
}

Keywords: Identification, Optimal Input Design, Recursive Estimation

\begin{abstract}
The problem of optimal input design for a specific fedbatch bioreactor case study is solved recursively. Hereto an adaptive receding horizon optimal control problem, involving the so-called E-criterion, is solved 'on-line', using the current estimate of the parameter vector $\theta$ at each sample instant $\left\{t_{k}, k=\right.$ $0, \ldots, N-h\}$, where $N$ marks the end of the experiment and $h$ is the control horizon for which the input design problem in solved. The optimal feed rate $F_{i n}^{\star}\left(t_{k}\right)$ thus obtained is applied and the observation $y\left(t_{k+1}\right)$ that becomes available is subsequently used in a recursive prediction error algorithm in order to find an improved estimate of the parameter estimate $\hat{\theta}\left(t_{k}\right)$. The case study involves an identification experiment with a Rapid Oxygen Demand TOXicity device for estimation of the biokinetic parameters $\mu_{\max }$ and $K_{S}$ in a Monod type of growth model. It is assumed that the dissolved oxygen probe is the only instrument available which is an important limitation. Satisfactory results are presented and compared to a 'naive' input design in which the system is driven by an independent binary random sequence with switching probability $p=0.5$. This comparison shows that, indeed, the optimal input design approach yields improved uncertainty bounds on the parameter estimates.
\end{abstract}

\section{Introduction}

The problem of optimal input design has received ample attention in the identification literature. Indeed, it is one of the classical identification problems that seeks to address an essential question for the model developer, namely whether it is possible to design a certain experiment in such a way that the parameters in the model structure can be uniquely identified and, moreover, how to design an input signal that minimizes (c.q. maximizes) an 'a priori' chosen norm of the Fisher information matrix (FIM) associated with the specific experimental setup and corresponding input signal. We shall not elaborate too extensively on the historical developments in this interesting field of study, but will only summarize some recent developments that have been reported on the subject. Our main goal in the current study is to focus on the optimal input design problem for a specific case study that seems to be relevant in the field of bioprocess control and, in addition, to introduce a new adaptive approach that solves the input design problem for a specific case study 'on-line', meaning that the best (current) estimate of the set of parameters $\theta$, i.e. $\hat{\theta}\left(t_{k}\right)$, is used to design an optimal input signal with respect to a cost criterion (including the FIM), after which the current estimate is 'optimally' updated to $\hat{\theta}\left(t_{k+1}\right)$ using a so-called recursive parameter estimation algorithm.

Recently, Stigter and Keesman have found analytical solutions for optimal input design signals with respect to one specific parameter $\theta_{i}$ in the model structure, [4, 8]. In this case the Fisher Information Matrix reduces to a scalar value which may be optimized using a singular control law that can be applied on a singular arc in state space that may be reached by a bang-bang control. The singular control law is derived by solving a set of algebraic equations, generated through repeated differentiation of the Pontryagin optimality condition $\frac{\mathrm{d} \mathcal{H}}{\mathrm{d} u} \equiv 0$ on the compact interval $\left[t_{1}, t_{2}\right]$, where $\mathcal{H}$ is the familiar Hamiltonian associated with the process model and a goal function that, essentially, depends on the parametric output sensitivities $\frac{\mathrm{d} y(t)}{\mathrm{d} \theta}$. The solution of this set of algebraic equations yields an explicit expression for the input function $u^{\star}(t)$. In addition, if the cost criterion involves the trace of the Fisher Information Matrix, i.e. the Acriterion, the input design problem may also be solved analytically and involves a maximization of the output sensitivities of a number of parameters for which an optimal input signal needs to be found.

Recent work of Versyck and co-workers includes the solution of the optimal input design problem with respect to the modified E criterion (see below) for several case studies using an approach in which the dynamic evolution of the sensitivity equations is analyzed in detail, [10]. This analysis is subsequently used to arrive at an optimal control problem that may be solved numerically, yielding an optimal switching time, initial substrate concentration, and substrate setpoint concentration for the a fedbatch reactor case study. A modified-E criterion of 1 is achieved for this experimental setup.

Although the reported analytical and numerical results are promising, their application in a practical case study involving a real experimental setup is still limited. This is especially the case in biochemical case studies where (i) the number of sensors is usually limited, meaning that not all the states can be measure directly, (ii) the sensors themselves may be very costly 
and (iii) may also involve high measurement uncertainties that deteriorate the parameter estimate $\hat{\theta}$ drawn from an experimental setup and corresponding input design. The search for more advanced algorithms that allow inclusion of these limitations is therefore a challenging problem that has an important practical significance. In addition, as to our knowledge the literature does not show an example in which the optimal input design problem is solved in tandem with a recursive parameter update scheme while this seems to be a natural choice since both problems involve the output parametric sensitivities to (i) arrive at the Fisher matrix for the experiment and (ii) to arrive at a gradient $\frac{\mathrm{d} y}{\mathrm{~d} \theta}$ pointing to a direction where a minimum of the innovations residuals (w.r.t. some norm) can be found. But let us first introduce some formal definitions in order to be able to be more specific in our discussion.

\subsection{Definitions}

Let the general, possibly non-linear, model structure $\mathcal{M}(\theta)$ for a specific system be given by

$$
\begin{aligned}
\frac{\mathrm{d} x(t)}{\mathrm{d} t} & =f(x(t), u(t), \theta) \\
y\left(t_{k}\right) & =h\left(x\left(t_{k}\right), u\left(t_{k}\right), \theta\right)+\eta\left(t_{k}\right)
\end{aligned}
$$

where $f: \mathbb{R}^{n} \times \mathbb{R}^{r} \times \mathbb{R}^{p} \rightarrow \mathbb{R}^{n}$ is a real valued vector function, i.e. the dynamical model $\mathcal{M}(\theta), h: \mathbb{R}^{n} \times \mathbb{R}^{r} \times \mathbb{R}^{p} \rightarrow \mathbb{R}^{m}$ is the observation (or read-out) equation, $\theta$ is a p-dimensional vector of time-invariant parameters, $x(t)$ is the $\mathrm{n}$-dimensional state vector, $u(t)$ is the r-dimensional input vector, and $y(t)$ is the m-dimensional output vector. We further assume that the observations $Y\left(t_{N}\right)=\left\{y\left(t_{k}\right), 0<k<N\right\}$, where $N$ marks the end of the experiment, is contaminated with a Gaussian white measurement noise process $\left\{\eta\left(t_{k}\right), 0<k<N\right\}$ with variance-covariance matrix $R$. Note that the above model definition is in continuous-discrete format which is a natural notation for physically based model, often used in biochemical and mechanical engineering.

\subsection{The Optimal Control Problem}

Since we wish to optimally identify the parameter set $\theta$ our control problem may be defined as follows

$$
\max _{u(t) \in \mathbb{U}_{a d m}} \phi\left(t_{f}\right)=\left\|\mathcal{F}\left(\theta, t_{f}\right)\right\|_{E}
$$

under the dynamical constraints (1), including the dynamical evolution of the local parametric output sensitivities $y_{\theta}(t) \triangleq$ $\frac{\mathrm{d} y(t)}{\mathrm{d} \theta}$ which can be derived straightforwardly from the model equations (1) and observation equations (2). Here, $\mathbb{U}_{a d m}$ is the set of admissible controls. In the above we have chosen, with some foresight into what follows, to use the so-called Ecriterion of the Fisher information matrix $(\mathcal{F})$, i.e. maximization of its minimal eigenvalue at the final time $t_{f}$. Other criteria such as D, modified E, or A-criterion, may be applied - each with its own advantages/disadvantages [6]. A useful alternative formulation of problem (3) is to consider the relative output sensitivities, appearing in the FIM, instead of the 'normal', un-weighted sensitivities, so that the sensitivities are scaled and relatively equal in size to one another.

Note that the cost function $\phi\left(t_{f}\right)$ in the above depends on the parameters $\theta$ whose values are not known 'a priori'. Let $u^{\star}\left(t_{k} \mid t_{k}\right)$ denote the optimal input calculated on basis of the estimate $\hat{\theta}\left(t_{k}\right)$, having processed the data record $Y\left(t_{k}\right)$. Then, a natural choice for a solution of the input design problem at time instant $t_{k}$ is to

1. Use the current estimate $\hat{\theta}\left(t_{k}\right)$ and solve the optimal input design problem (3) over a time horizon $\left[t_{k}, t_{k+h}\right]$,

2. Apply the 'optimal' input $u^{\star}\left(t_{k} \mid t_{k}\right)$ thus obtained on the interval $\left[t_{k}, t_{k+1}\right)$, assuming for simplicity a zero-orderhold mechanism for the input signal $u^{\star}\left(t_{k} \mid t_{k}\right)$,

3. Sample the system at time instant $t_{k+1}$, thereby obtaining a new measurement $y\left(t_{k+1}\right)$,

4. Process the new measurement to find an improved value of the current estimate $\hat{\theta}\left(t_{k}\right)$, yielding $\hat{\theta}\left(t_{k+1}\right)$, and repeat the procedure using $\hat{\theta}\left(t_{k+1}\right)$.

In summary, our approach is to solve the above identification problem as an adaptive receding horizon optimal control problem that processes the parameter estimates recursively each time an observation $y\left(t_{k+1}\right)$ becomes available.

Our choice for the recursive parameter reconstruction algorithm to update the estimate $\hat{\theta}\left(t_{k}\right)$ in step (4) in the above, is a so-called 'recursive prediction error' algorithm in continuousdiscrete format, [5, 7]. The advantage of this specific algorithm is that it includes the parametric sensitivities of the states, i.e. $W(t) \triangleq \frac{\mathrm{d} x(t, \hat{\theta})}{\mathrm{d} \theta}$, in a natural way as to arrive at an estimate of the gradient $\frac{\mathrm{d} y(t, \hat{\theta})}{\mathrm{d} \theta}$ which is minimized with respect to the prediction error of the prediction $\hat{y}\left(t_{k+1}, \theta\right)$. The algorithm achieves this minimization through calculation and subsequent interpretation of the innovation

$$
\epsilon\left(t_{k}\right)=y\left(t_{k}\right)-\hat{y}\left(t_{k}, \hat{\theta}\left(t_{k}\right)\right)
$$

which is, loosely speaking, the mismatch of the last model prediction $\hat{y}\left(t_{k}, \hat{\theta}\left(t_{k}\right)\right)$ w.r.t. the current sample $y\left(t_{k}\right)$. The interpretation of the innovation is performed through calculation of a gain matrix which is assumed at steady state for the state vector and is calculated explicitly on the basis of a variancecovariance matrix $P_{\theta \theta}\left(t_{k}\right)$ for the estimated parameters in the model structure. Since the parametric sensitivities $W(t)$, a $(n \times p)$ matrix, become available once the receding horizon optimal control problem is solved at time instant $t_{k}$ (step (1) in the above procedure), these sensitivities can be used immediately for an update of the parameter vector $\hat{\theta}\left(t_{k}\right)$. Finally, it should also be mentioned that the recursive prediction error framework provides a unifying perspective on the problem of optimal input design in tandem with recursive parameter estimation since both algorithms use the same sensitivity functions - each, however, for its own goal. 


\section{The Case Study}

\subsection{A Dynamical Model for Oxygen Uptake in a Fedbatch Bioreactor Setup}

The case study we wish to address here has been defined in the work by Vanrolleghem, [9]: For determination of the biokinetic parameters $\mu_{\max }$ and $K_{S}$ an identification experiment with a so called Rapid Oxygen Demand TOXicity Device (RODTOX) can be conducted and respirometic data can be obtained. Generally speaking, the respirogram characterizes the healthy state of a biomass concentration $\left(C_{X}(t)\right)$ and may be used, for example, to identify a toxic alarm, i.e. the respirometric signature obtained with a RODTOX may be 'non-standard', meaning that the biomass does not perform normally due to the presence of a toxic in the feeding substrate $\left(C_{S}(t)\right)$.

The biokinetic model for the RODTOX device may be presented in a more general context, i.e. as a fedbatch reactor in which substrate (including dissolved oxygen) is fed into the reactor with a feedrate $F_{i n}(t)$, instead of 'only' one initial impulse substrate concentration at the very beginning of the experiment. We assume that the dissolved oxygen concentration in the feeding substrate is at saturation level, i.e. it is not affected by the solute nor the presence of bacteria which are assumed not to be present in the feeding substrate. The consumption of substrate by the bacteria in the reactor is aerobic and directly affects the dissolved oxygen concentration in the vessel. A dynamic model of the process reads:

$$
\begin{aligned}
& \frac{\mathrm{d} C_{D O}(t)}{\mathrm{d} t}=k_{L a}\left(C_{s a t}^{e n}-C_{D O}(t)\right)-O U R(t)+\cdots \\
& \frac{F_{\text {in }}(t)}{V(t)}\left(C_{s a t}-C_{D O}(t)\right) \\
& \frac{\mathrm{d} C_{X}(t)}{\mathrm{d} t}=\mu\left(C_{S}(t)\right) C_{X}(t)-\frac{F_{i n}(t)}{V(t)} C_{X}(t) \\
& \frac{\mathrm{d} C_{S}(t)}{\mathrm{d} t}=-\frac{\mu\left(C_{S}(t)\right)}{Y} C_{X}(t)+\cdots \\
& \frac{F_{i n}(t)}{V(t)}\left(C_{S i n}-C_{S}(t)\right) \\
& \frac{\mathrm{d} V(t)}{\mathrm{d} t}=F_{\text {in }}(t) \\
& O U R(t)=(1-Y) \frac{\mu\left(C_{S}(t)\right)}{Y} C_{X}(t) \\
& \mu\left(C_{S}(t)\right)=\frac{\mu_{\max } C_{S}(t)}{K_{S}+C_{S}(t)} \\
& y\left(t_{k}\right)=\left(\begin{array}{lll}
1 & 0 & 0
\end{array}\right)\left(\begin{array}{c}
C_{D O}\left(t_{k}\right) \\
C_{X}\left(t_{k}\right) \\
C_{S}\left(t_{k}\right)
\end{array}\right)
\end{aligned}
$$

where $k_{L a}$ is the re-aeration constant [1/min], $V(t)$ is the volume of the solvent [L], including biomass and substrate, $C_{s a t}^{e n}$ is the saturation concentration of dissolved oxygen, including a small (constant) correction for the endogenous respiration of the biomass $[\mathrm{mg} / \mathrm{L}], C_{s a t}$ is the (normal) saturation concentration of dissolved oxygen $[\mathrm{mg} / \mathrm{L}], \mu_{\max }$ is the maximum specific growth rate $[\mathrm{mg} / \mathrm{min}], K_{S}$ is the saturation constant $[\mathrm{mg} / \mathrm{L}], Y$ is the yield coefficient of biomass on substrate
$\left[\mathrm{mg}\left(C_{X}\right) / \mathrm{mg}\left(C_{S}\right)\right]$, and $O U R(t)$ is the oxygen uptake rate of the biomass in the reactor $[\mathrm{mg} / \mathrm{L} . \mathrm{min}]$. Note that in the above model it is assumed that there are dissolved oxygen data only and no biomass concentration data, nor substrate data, nor oxygen uptake rate data which would require differentiation of the dissolved oxygen data w.r.t. time and, therefore, would be sensitive to high frequencies in the measurement noise process $\eta\left(t_{k}\right)$. The limited sensor availability causes the problem of reconstructing $\mu_{\max }$ and $K_{S}$ to be badly defined. Moreover, the reconstruction of these parameters from noise corrupted data are hampered because of the same measurement noise process and, although the two parameters are theoretically identifiable, practical identification from these data is indeed very difficult [3]. At the root of this persistent problem lies a correlation of the parametric sensitivities $y_{\mu_{\max }}(t) \triangleq \frac{\mathrm{d} y(t)}{\mathrm{d} \mu_{\max }}$ and $y_{K_{S}}(t) \triangleq \frac{\mathrm{d} y(t)}{\mathrm{d} K_{S}}$. In the sequel we will focus on the combined estimation of $\mu_{\max }$ and $K_{S}$ and we will assume that the parameters $Y$ and $k_{L a}$ have been estimated off-line or have been established via the abundantly available literature. Both parameters can be specified relatively easily in comparison with the parameter combination $\left\{\mu_{\max }, K_{S}\right\}$.

\section{Results}

In a numerical experiment the true values for the parameters were set to the values summarized in tables 1 and 2 . The goal function to be minimized was chosen to be the E-criterion, meaning that maximization of the minimum eigenvalue of $\mathcal{F}\left(t_{f}\right)$ was desired. Other criteria, such as the modified-E criterion and the D-criterion were considered too but it appeared that especially the modified-E criterion was extremely difficult too achieve. Between the E-criterion and D-criterion, the best results were obtained using the former and these results will therefore be presented here. An important variable in the numerical experiment is the control horizon $h$, whose value influences the 'smoothness' of the optimal solution $u^{\star}\left(t_{k} \mid t_{k}\right)$ on the prediction-horizon interval $\left[t_{k}, t_{k+h}\right]$. A workable value appeared to be $h=4$ minutes. Since the discretisation variable $\Delta t=t_{k+1}-t_{k}$ was set to 10 seconds, the optimal control algorithm optimized 24 constant input values $\left\{u\left(t_{k}^{i} \mid t_{k}\right), i=\right.$ $1, \ldots, 24\}$ on the interval $\left[t_{k}, t_{k+h}\right]$. As said before, this optimization was performed each time after the next dissolved oxygen observation $y\left(t_{k+1}\right)$ was processed by the recursive prediction error algorithm in order to improve the estimate $\hat{\theta}\left(t_{k}\right)$. Clearly, the computational burden can become intense if a finer discretisation grid or larger control horizon is chosen 'a priori'. The presented values for $h$ and $\Delta t$, however, turned out to give 'manageable' CPU times on a personal computer platform. The observations $y\left(t_{k}\right)$ were generated 'on-line' through simulation of the true system, after which a measurement noise with a variance $R=0.01$ was added to the simulated values.

In order to make a good comparison of the adaptive optimal input design solution this case was compared with an identical case, but now with a random binary input sequence with a switching probability $p=0.5$. Implementation of this 


\begin{tabular}{|c|c|c|}
\hline & $\bar{\theta}$ & $\hat{\theta}(0)$ \\
\hline$\mu_{\max }$ & $2.62 \cdot 10^{-4}$ & $5.0 \cdot 10^{-4}$ \\
\hline$K_{S}$ & 1.0 & 0.5 \\
\hline$Y$ & 0.64 & N/A \\
\hline
\end{tabular}

Table 1: Biokinetic parameter values after [9] and initial estimates for these parameters in the two optimal input design studies reported here.

\begin{tabular}{|c|c|c|}
\hline$C_{S}(0)$ & $C_{D O}(0)$ & $C_{X}(0)$ \\
\hline 0.0 & 7.0 & 4000 \\
\hline
\end{tabular}

Table 2: Initial conditions for the specific setup.

sequence causes the substrate feedrate pump to switch from $F_{\text {in }}(t)=0$ to $F_{\text {in }}(t)=F_{\max }=0.2 \mathrm{~L} / \mathrm{min}$ at random on the switching times $\left\{t_{k}, k=0, \ldots, N\right\}$ (see figure 1 , bottom).

Figure 1 shows the adaptive optimal receding horizon inputs for both cases. It is interesting to see that the optimal result is to inject a pulse of substrate into the reactor at approximately 3.2 minutes, after the recursive estimator has already learned from the initial 'modest' feeding fase where the feedrate is only small. This pulse causes the uncertainty in especially the estimate of $\mu_{\max }$ to decrease substantially, as desired (figure 2 , top-left). Note also that the tail of the optimal input signal $u^{\star}\left(t_{k}\right)$ exhibits slight 'ringing' behavior, causing the feedpump to add small pulses of substrate which, on average, represent an almost constant, small feedrate. These small pulses are added after the biomass has settled in a short zero feeding fase, just after the large pulse of substrate. It is also interesting to see that the uncertainty bounds on the parameter $K_{S}$ are much smaller in the adaptive optimal receding horizon case than in the case of a random binary input sequence (figure 2, right). Apparently, the random binary sequence is not sufficiently exciting the dynamics in the bioreactor in order to deduce a reliable estimate of the saturation concentration parameter. This confirms the well-known fact that estimation of the parameter $K_{S}$ from oxygen (uptake) data is difficult in practice and requires a careful design.

Figure 3 shows both the unobserved and observed state estimates generated by the recursive prediction error algorithm. Clearly, the algorithm is capable of tracking the dissolved oxygen dynamics in the bioreactor sufficiently well. The unobserved states were also found to be reliable reconstructions. The optimal input sequence $u^{\star}\left(t_{k} \mid t_{k}\right)$ causes the dissolved oxygen state to settle down in an almost steady state after the increased oxygen demand due to the substrate pulse at $3.2 \mathrm{~min}$ utes has decayed away. In this steady state the recursive estimator is only learning at a small rate, since the uncertainty bounds on the parameter estimates do not decrease substantially after approximately 8 minutes (figure 2).

Finally, a good indicator for the information content of the conducted experiment is to inspect the trajectories of the Fisher
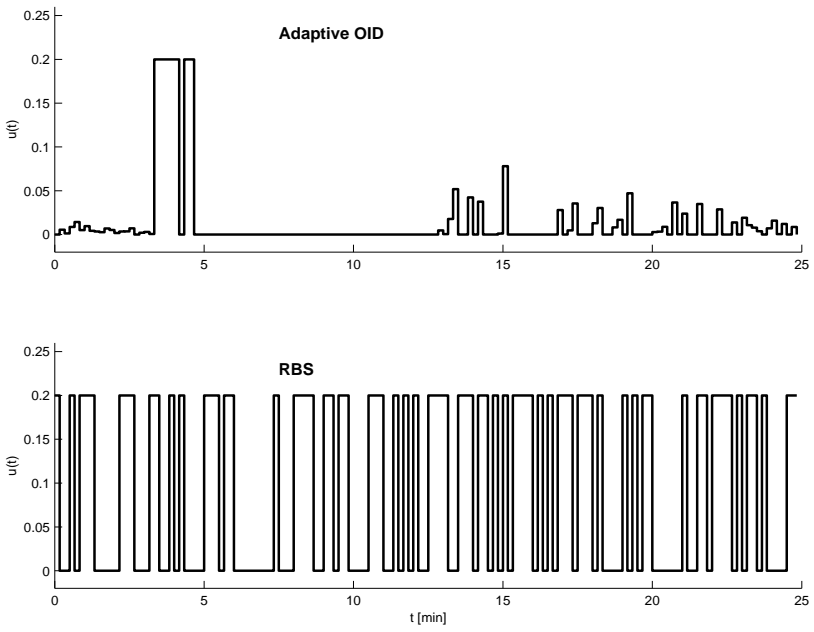

Figure 1: Optimal adaptive receding horizon input (top) and random binary sequence input (bottom).
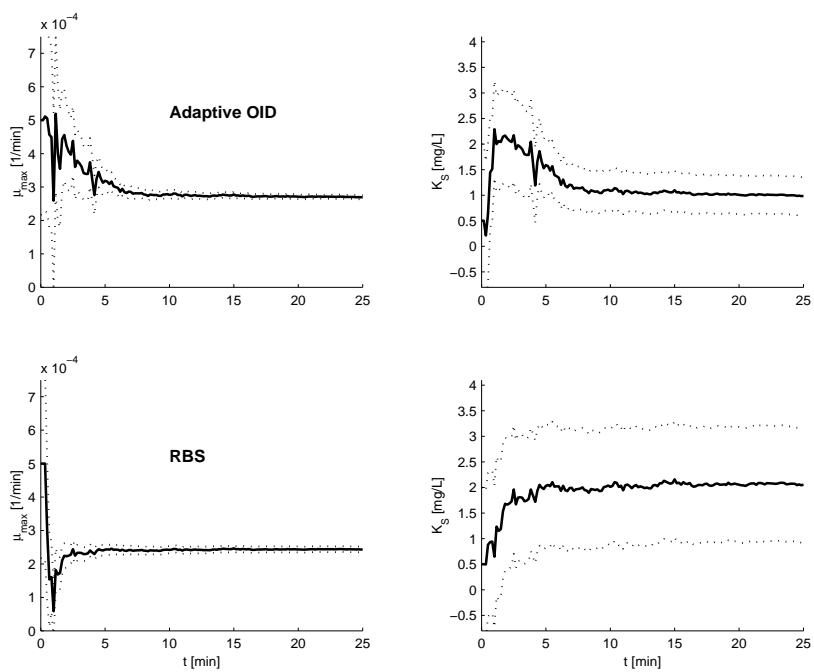

Figure 2: Optimal adaptive receding horizon estimates, generated by a recursive prediction error algorithm (top) and recursive parameter estimates for the random binary input sequence (bottom).

Information Matrix elements $\left\{f_{i j}(t), i, j=1,2\right\}$. Figure 4 presents these trajectories, including the evolution (in time) of the goal function (E-criterion) for the two cases of adaptive optimal receding horizon control and random binary sequence control. Clearly, the information content for especially the parameter $K_{S}$, corresponding to $f_{22}(t)$ in figure 4 , substantially increases if the input is carefully designed using an E-criterion. This is confirmed after calculating the corresponding ellipsoids for the parameter estimate $\hat{\mu}_{\max }$ and $\hat{K}_{S}$ at time $t_{0}$ and time $t_{f}$ (see figure 5). Maximization of the E-criterion causes the volume of the ellipsoid to increase substantially in comparison to a 'naive' random binary sequence input design and the adaptive optimal input design is therefore to be preferred. 

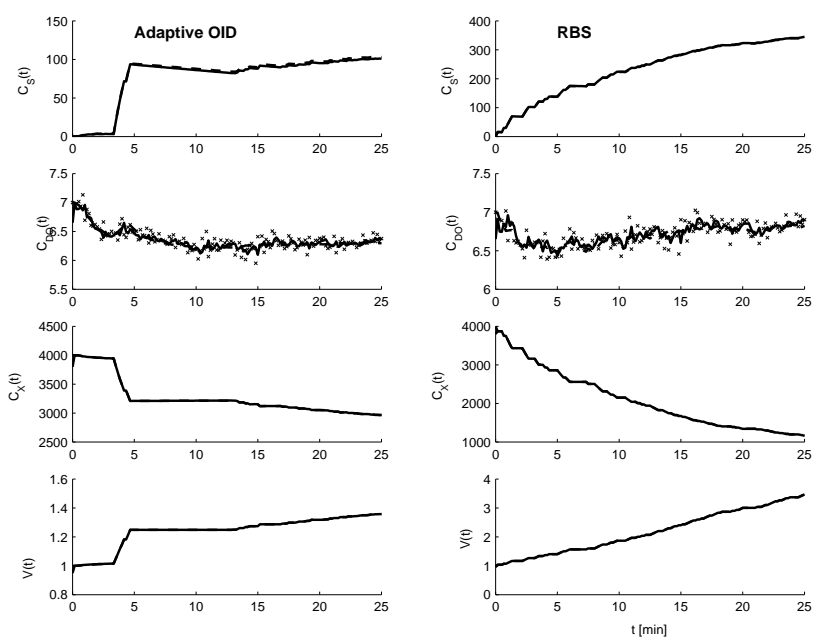

Figure 3: State estimates (unmeasured signals $C_{X}(t), C_{S}(t)$, and $V(t)$ and measured signal $C_{D O}(t)$ ), generated by the recursive prediction error algorithm. True and estimated states were both plotted and can hardly be discerned.
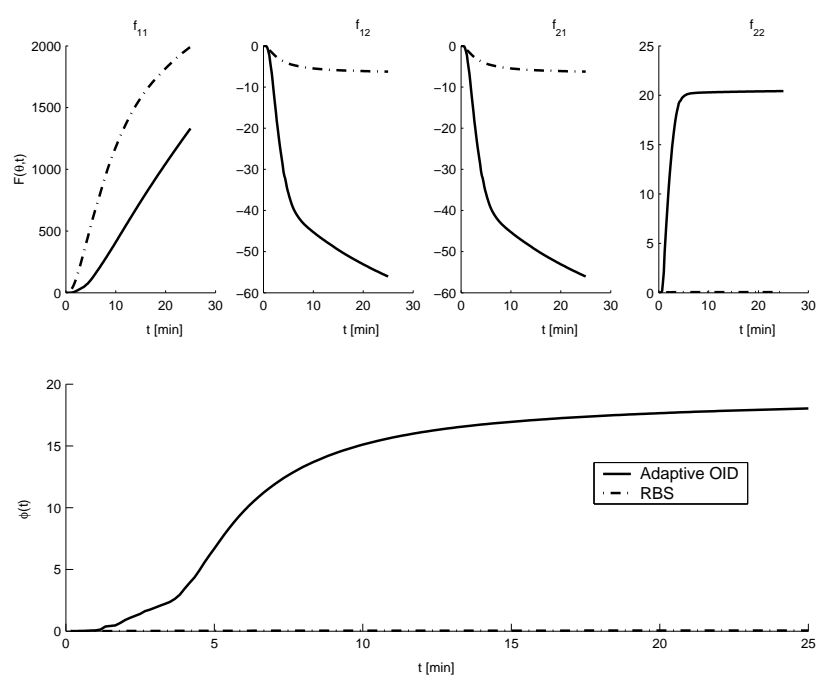

Figure 4: Fisher Information Matrix element trajectories $f_{11}(t), f_{12}(t), f_{21}(t)$, and $f_{22}(t)$ (top) and the E-criterion objective function (bottom) for both cases.

\section{Conclusions}

An optimal adaptive receding horizon optimal control problem was solved using a (direct) optimal control algorithm in tandem with a recursive prediction error algorithm. The specific fedbatch case study shows that the combination of these two algorithms yields satisfactory results that can be implemented 'on-line'. The specific solution obtained here includes a small feeding fase, followed by a substrate pulse, after which the feedrate is switch off for a short time interval and continues at a small constant feedrate. It was argued that especially when the experiments are costly and involve a limited number of sensors, the approach may show very useful.

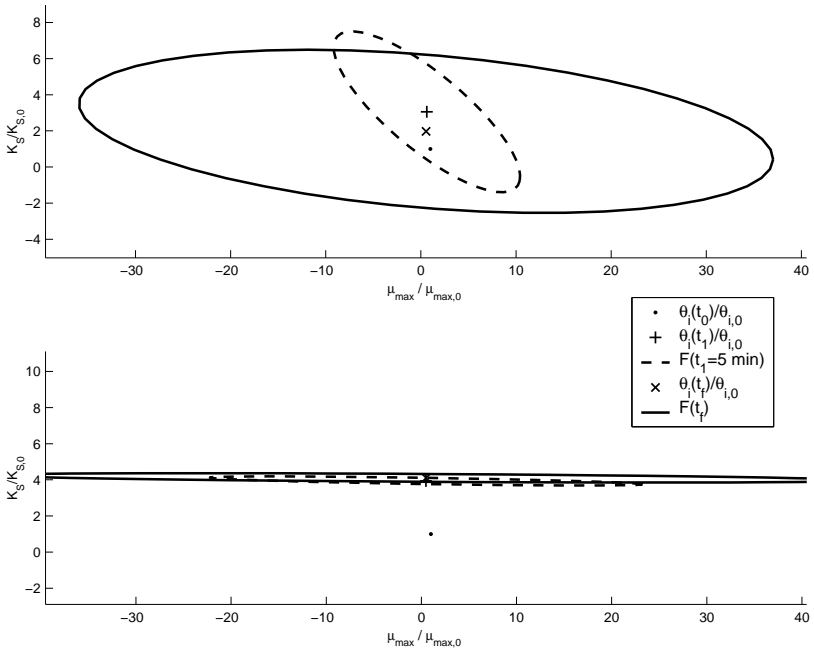

Figure 5: Ellipsoids at both an initial $\left(t_{0}=5 \mathrm{~min}\right)$ and final time $\left(t_{f}=25 \mathrm{~min}\right)$ of the experiment for the adaptive receding horizon case (top) and the random binary sequence case (bottom). Clearly, the adaptive receding horizon results are superior to the random-binary-sequence experiment.

Generally speaking, first principle models, based on a sound physical foundation, are vehicles of our hypotheses and, as such, are rewarding because they provide insight into the process under study. It is well known that it is increasingly difficult to derive these type of models if the process is complex and exhibits a myriad of interactions between numerous states and their associated parameters. In fact, the growth model considered in our case study may be regarded as 'black-box' and it is considered an archetype model that has shown its value in many instances. It is important to focus on optimal input design studies as presented in the current study since the search for minimal uncertainty bounds on the parameters in the model structure, obtained via the input/output data record, increases the validity, or otherwise, of the model structure as a whole for both physically based and 'black-box' type of models, [1]. Optimal input design tools may therefore become very useful in the search for a satisfactory model for a complex biochemical system such as presented here or, for example, in the classical BOD-DO interaction model, [2]. Although not all these systems may be controlled freely at will, meaning essentially that the set $\mathbb{U}_{a d m}$ can indeed be very small, one could progress similar to the avenue taken by Vanrolleghem where a small pilot plant was constructed that allows a broader range of admissible input signals and subsequent excitations, [9]. In these cases an adaptive optimal input design algorithm may be applied, not only to estimate the values of the parameters in the model structure, but to assess the validity of the model as a whole.

\section{References}

[1] M. B. Beck, J. D. Stigter, and D. Lloyd-Smith. Elastoplastic Deformation of Structure. In M. B. Beck, editor, Environmental Foresight and Models: A Manifesto, pages 
323-350. Elsevier, Oxford, 2002.

[2] M. B. Beck and P. C. Young. Systematic Identification of DO-BOD Model Structure. Journal of the Environmental Engineering Division, 102, EE5:902-927, 1976.

[3] A. Holmberg and J. Ranta. Procedures for Parameter and State Estimation of Microbial Growth Process Models. Automatica, 18(2):181-193, 1982.

[4] K. J. Keesman and J. D. Stigter. Optimal parametric sensitivity control for the estimation of kinetic parameters in bioreactors. Mathematical Biosciences, 179:95-111, 2002.

[5] L. Ljung and T. Söderström. Theory and Practice of Recursive Identification. M.I.T. Press, Massachusetts, 1983.

[6] A. Munack. Optimal feeding strategy for identification of monod-type models by fed-batch experiments. In N. M. Fish, editor, Computer Applications in Fermentation Techology, Modelling and Control of Biotechnological Processes, pages 195-204. Elsevier, Amsterdam, 1989.

[7] J. D. Stigter. The Development and Application of a Continuous-Discrete Recursive Prediction Error Algorithm in Environmental Systems Analysis. $\mathrm{PhD}$ thesis, University of Georgia, Athens (GA), USA, 1997.

[8] J. D. Stigter and K. J. Keesman. Optimal parametric sensitivity control of a fed batch reactor. In Proceedings European Control Conference 2001, Porto, Portugal, pages 2841-2844, 2001.

[9] P. A. Vanrolleghem and M. Van Daele. Optimal experimental design for structure characterization of biodegradation models: On-line implementation in a respirographic biosensor. Water Science and Technology, 30(4):243-253, 1994.

[10] K. J. Versyck. Dynamic Input Design for Optimal Estimation of Kinetic Parameters in Bioprocess Models. PhD thesis, Katholieke Universiteit Leuven, 2000. 